\title{
Strain and Curvature Stability Enhanced SMF Introduction
}

\author{
Somayeh Makouei \\ Faculty of Electrical and Computer Engineering, University of Tabriz, Tabriz 51664, Iran \\ E-mail: makouei@tabrizu.ac.ir
}

\begin{abstract}
In this paper, the strain insensitive single-mode optical fiber with low nonlinear effects and ultra low bending loss (BL), appropriate for small curvature radius installation, is presented. The suggested design method is based on electrical field distribution Gaussian assumption in the core region which evaluates the refractive index profile considering proper mode field diameter (MFD) value. Then, so as to attain the enhanced bending loss and strain stability response for the optical fiber, the optimization tool of the evolutionary genetic algorithm (GA) is employed to determine the optical and geometrical parameters of the structure. In the first designed fiber, the calculations for BL, MFD, effective area $\left(A_{\text {eff }}\right)$, and effective refractive index sensitivity to $\operatorname{strain}\left(\mathrm{dn}_{\mathrm{eff}} / \mathrm{d} \varepsilon\right)$ in the well-known wavelength of $1.55 \mu \mathrm{m}$ are $0.0018 \mathrm{~dB}$ per each turn of $5 \mathrm{~mm}$ curvature radius, $8.53 \mu \mathrm{m}, 58 \mu \mathrm{m}^{2}$, and $-4.5 \times 10^{-8} \mu \varepsilon^{-1}$, respectively. Furthermore, the effect of placing raised outer cladding in the fiber structure is investigated which exhibits the MFD of $8.63 \mu \mathrm{m}$, the bending loss of $0.0093 \mathrm{~dB}$ for single turn of 5 $\mathrm{mm}$ radius, and the $\mathrm{A}_{\text {eff }}$ of $87 \mu \mathrm{m}^{2}$ at $1.55 \mu \mathrm{m}$. In this case the strain sensitivity of $n_{\text {eff }}$ is reached to $-6.7 \times 10^{-8} \mu \varepsilon^{-1}$. The mentioned effective area is magnificently large in the domain of bend insensitive fibers. In the meantime, the designed structures are insensitive to strain which is a crucial feature in applications with small curvature radius.
\end{abstract}

\section{Introduction}

Current strong demands on computer communication services like internet access services require economical solutions to provide broadband capability to access networks. The broadest broadband is optical fiber; the only medium capable of moving data at multi-gigabit-per-second speeds. These demands lead the optical fiber the suitable choice for home applications [1, 2]. In order to rival conventional non-zero dispersion shifted fiber based system, the designed optical fiber must be efficient, commercially. However, apart from the economical option, several design considerations arise in the home applications [3]. The first challenge is to reduce the bending loss to meet the requirements of harsh end copper cable-like handling conditions. Bending loss in optical fibers has been studied, extensively, chiefly because of its adverse effect on power budget in telecommunications. The optical fiber is compelled to be bent at various corners of walls and the bends can be as small as $5 \mathrm{~mm}$, and hence a severe power penalty is caused. Bending loss of less than $0.1 \mathrm{~dB} /$ turn will ensure robust network performance under practical bending conditions such as tight ninety degree corners, fixation by stapling and excess cable storage in tightly confined spaces. Design of bend insensitive fibers for home application includes important technical challenges. The second challenge is the undesirable induced strain due to holders and staples in the small radius installations. The transmission characteristics of the designed bend insensitive optical fiber must be resistant to the refractive index profile variations made by the external applied strain $[4,5]$. The next challenge is compatibility with the standard singlemode fibers (SMFs) installed in the telecommunication industry. This compatibility could be achieved by reducing splicing loss during splicing of bend insensitive fiber structures with the conventional SMF [6]. This is attained through adjustment of the MFD of the designed fiber to that of the standard conventional fiber.

There are several methods to reduce the bending loss. The simplest way to reduce the bending loss of a fiber is to increase the refractive index difference between the core and cladding in the step index profile [7]. This way results in MFD reduction. Furthermore, higher order modes would be appeared in it. Other bend minimizing techniques include depressed cladding, adding a low index trench, and photonic crystal fiber employment [8-10]. Photonic crystal fibers (PCFs) have shown superior transmission performance but their fabrication process is much more complicated than conventional fiber manufacture process. In contrast to conventional optical fibers, in PCFs, not only in higher wavelengths the BL surpasses the critical value but also in lower wavelengths they hold a short-wavelength bend loss edge due to bend-induced coupling from the fundamental to the higher-order modes [11].

Considering the three mentioned challenges, it is endeavored to introduce a design procedure so as to overcome these problems and present an optical fiber, holding superior characteristics in case of BL and nonlinear effects, without any reduction in MFD. Note that this structure is capable of use in small curvature applications, since the fiber response to any induced external strain is perfectly optimized.

The paper organization is as follows.

The design procedure and mathematical relations are presented in section 2 . Then, in section 3 , simulation results 
are discussed for validating our suggested process. The multilayer cladding impact is exhibited in section 4. Finally, the paper ends up with a brief conclusion.

\section{Mathematical formulation and design procedure}

The purpose of this paper is introduction of a design method to evaluate the profile for refractive index of the optical fiber with suitable resistance against small radius curvature and induced external strain by controlling the mode field diameter, straightly. The procedure is based on the reverse engineering. Firstly, a relevant function for the modal field distribution is assumed. Through some logical considerations, the suitable refractive index profile holding the desired MFD is extracted. In the weakly guiding approximation, the transverse component of the electric field satisfies the scalar wave equation [6]:

$r^{2} \frac{d^{2} \Psi(r)}{d r^{2}}+r \frac{d \Psi(r)}{d r}+\left\{k_{0}^{2}\left[n^{2}(r)-n_{e f f}^{2}\right] r^{2}-l^{2}\right\} \Psi(r)=0$

where, $\Psi(r)$ is the electric field along radius $r, k_{0}$ is the wave number in the free space, $n(r)$ is the refractive index profile of fiber, $l$ is the azimuthal mode number and $n_{\text {eff }}$ is the effective refractive index of the structure.

The Gaussian approximation of the fundamental mode in conventional optical fibers gives this idea to choose exact Gaussian function as an answer of wave equation in the core layer [12]. In this case the relationship between $n_{\text {eff }}$ and MFD is available at exclusive operational wavelength $\left(\lambda_{\text {op }}\right)$. However, similar to all kinds of optical fibers, the refractive index of least cladding layer must be constant, so the assumption of normalized electrical field distribution is extended to the following model:

$\left.\Psi(r)\right|_{\lambda_{o p}}= \begin{cases}e^{\frac{-r^{2}}{\omega^{2}}} & r<r_{e} \\ A_{e} K_{l}\left(\gamma_{e} r\right) & r>r_{e}\end{cases}$

where $\omega=\mathrm{MFD} / 2, r_{e}$ and $K_{l}$ are core radius and modified Bessel function of the $l$ order. $A_{e}$ and $\gamma_{e}$ are defined as follows:

$A_{e}=\frac{\exp \left(\frac{-r_{e}^{2}}{\omega^{2}}\right)}{K_{l}\left(\gamma_{e} r_{e}\right)}, \gamma_{e}=k_{0} \sqrt{n_{e f f}^{2}-n_{e}^{2}}$

where $n e$ is the clad refractive index. This electrical field distribution must satisfy the Eq. 1 at $\lambda_{\text {op }}$ wavelength. To avoid any sharp difference in the refractive indexes of the core and the clad, the refractive index of the clad layer $\left(n_{\mathrm{e}}\right)$ must be equal with the core refractive index at the core and clad boundary (re). This subject is not mentioned in $[13,14]$. Straightaway, the profile of the refractive index is extracted. By substituting Eq. 2 in Eq. 1:

$n(r)= \begin{cases}\sqrt{n_{e f f}^{2}+\frac{4}{k_{0}^{2} \omega^{2}}\left(1-\frac{r^{2}}{\omega^{2}}\right)}, & r \leq r_{e} \\ \sqrt{n_{e f f}^{2}+\frac{4}{k_{0}^{2} \omega^{2}}\left(1-\frac{r_{e}^{2}}{\omega^{2}}\right)}, & r>r_{e}\end{cases}$

Based on the mentioned equation, the refractive index profile directly depends on the values of the MFD, core radius, and effective refractive index. In the meantime, the type of the core refractive index is graded and peaks in the core center. By substituting the special definition of the $n e$ in Eq. 3, the $\gamma_{e}$ is defined as follows: $\gamma_{e}=\frac{2}{\omega} \sqrt{\left(\frac{r_{e}^{2}}{\omega^{2}}-1\right)}$.

In order to guarantee the single-mode operation, the modal analyses of estimated graded index optical fiber is done by piecewise constant representation of the graded index profile that resembles a staircase. In this approach, the refractive index of optical fibers with an arbitrary but axially symmetric profile is approximately expressed by a staircase function. According to the linear polarization approximation (LP), the guided modes and propagating wave vectors can be obtained by using transfer matrix method (TMM) [15].

After the extraction of the refractive index profile with the desired mode field diameter, the bending loss and strain response of the fiber is taken into account. In single-mode fibers, the radiation loss from the fundamental mode to leaky core modes arises whenever there is a change in curvature of the fiber axis. Using the method introduced and discussed by Jun-ichi Sakai et al. [16], the radiation loss, owing to the uniform bending can be obtained. In this method, it is supposed that the field near the inner layers in the curved fiber is almost similar to that in the straight one. This approximation is greatly accurate to evaluate the radiation losses in single-mode optical fiber [17]. The radiation loss $2 \alpha$, due to uniform bending, is defined by the following equation:

$2 \alpha=\frac{\sqrt{\pi} A_{e}^{2}}{4 P} \times \frac{r_{e} \exp \left(\frac{-4 \Delta w^{3}}{3 r_{e} v^{2}} R_{b}\right)}{w\left(\frac{w R_{b}}{r_{e}}+\frac{v^{2}}{2 \Delta w}\right)^{0.5}}$

$P=\int_{0}^{\infty} \Psi(r)^{2} r d r$

which in $r_{e}$ denotes the core and least cladding boundary position, referring to Eq. 2, $A_{e}$ is the constant component of the electrical field of the outer layer, $R_{b}$ is the radius of curvature of the bend, $w$ is given by $\left(r_{e} \cdot \gamma_{e}\right)$ and $\Delta$ is the refractive index difference between $n_{\max }$ and $n_{e} . v$ is the normalized frequency which is defined by:

$v=k_{0} r_{e} \sqrt{n_{\max }^{2}-n_{e}^{2}}$.

The $n_{\max }$ is happened at the core center which is defined as follow:

$n_{\max }=\sqrt{n_{e f f}^{2}+\frac{4}{k_{0}^{2} \omega^{2}}}$.

The optical fiber response to strain occurs due to changes in the refractive index and physical dimensions of the layer, both of which manage the $n_{\text {eff }}$ variation [18]. Physical change in core height and width is related to the Poisson ratio $(v)$. The term relating the refractive index changing to strain is dominated by photoelastic effect. Getting exposed to strain, the bandgap shift of silica causes the change in the absorption coefficient of it. The variation in the absorption coefficient affects the refractive index of silica. Therefore, the strain-induced bandgap change is related to the changes of the refractive index [4]. Generally, the change in refractive index is deduced to be [5]

$$
\Delta n_{i}=\sum_{i, j=1}^{6} \frac{\partial n_{i}}{\partial \varepsilon_{j}} \varepsilon_{j}+\frac{\partial n_{i}}{\partial T} \Delta T=-\frac{n_{i}^{3}}{2} \sum_{i, j=1}^{6} p_{i j} \varepsilon_{j}+\frac{\partial n_{i}}{\partial T} \Delta T
$$

Where $p_{i j}$ are the photoelastic coefficients, $n_{i}$ is the refractive index of the $i t h$ layer, $\varepsilon_{j}$ is the strain tensor element and $\Delta T$ is the temperature variation. Assuming 
the homogeneity and isotropic behavior of silica and also the application of force perpendicular to the fiber cross section, Eq. 9 can be simplified to

$\Delta n_{i}=-\frac{n_{i}^{3}}{2}\left(p_{12}-v\left(p_{11}+p_{12}\right)\right) \varepsilon+\frac{\partial n_{i}}{\partial T} \Delta T$

Where, for the pure silica at $1550 \mathrm{~nm}, p_{11}=0.113, p_{12}=0.252$, $v=0.17$.

Accomplishing the presentation of the prerequisite relevant relations, the optimization of the bending loss and strain behaviors of the structure is regarded. In order to design the bend insensitive optical fiber with a satisfactory resistance to external induced strain, the GA is employed [19]. In this process, the optical and geometrical parameters of the fiber are set by minimization of the appropriate cost function at operational wavelength which is defined as follows:

cost function $\left.\right|_{\lambda_{o p}}=\left.\alpha B L\right|_{\lambda_{o p}}+\left.\gamma S_{\varepsilon}\right|_{\lambda_{o p}}$,

$S_{\varepsilon}=\frac{\Delta n_{e f f}}{\Delta \varepsilon}$

Where $\varepsilon$ is the unavoidable induced strain in installation processes with small curvature radius and $\alpha$ and $\gamma$ are the weight coefficients which prohibit the dissipation of the low value term in the cost function confronting the high value term. Since $n_{\text {eff }}$ stands for the most fundamental feature of the optical fiber, its variation as a result of strain induction switches the fiber characteristics. So, the less sensitivity of $n_{\text {eff }}$ to strain assures the more stability of structure response.

\section{Simulation results and discussion}

Based on the procedure explained in the previous section, the proposal of the bend and strain insensitive single-mode optical fiber is presented. All the results exhibited in this section are attained at $\lambda_{\text {op }}=1.55 \mu \mathrm{m}$, bend radius of $5 \mathrm{~mm}$ and the desired MFD of $8.5 \mu \mathrm{m}$. Moreover, the induced strain is considered to be in $\pm 10000 \mu \varepsilon$ interval. During the simulations the room temperature is preserved. The distinct pairs of $n_{\text {eff }}$ and $r_{e}$ form the forty chromosome initial population in GA.

The outputs of the optimization tool are listed in Table 1. It is obvious that the core radius of the designed structure is large which is good from fabrication point of view.

Table 1: GA results for optimal parameters value.

\begin{tabular}{|c|c|c|}
\hline Parameter & $\mathrm{n}_{\mathrm{eff}}$ & $\mathrm{r}_{\mathrm{e}}(\mu \mathrm{m})$ \\
\hline Value & 1.4501 & 4.802 \\
\hline
\end{tabular}

The refractive index profile for the designed bend and strain insensitive single-mode optical fiber is illustrated in Figure 1. This profile is calculated by adjustment of the mode field diameter value to $8.5 \mu \mathrm{m}$ at $1.55 \mu \mathrm{m}$ operational wavelength. The conventional approach for bending loss reduction in the optical fibers is the shrinkage of the MFD by the means of augmentation of the difference in the refractive indices of the core and the cladding which may lead to high inequality in viscosity properties of the layers. As a result, extra mechanical and thermal stresses are produced in the fiber [20,21]. The graded nature of the refractive index profile in the core region would reduce the outline stresses.

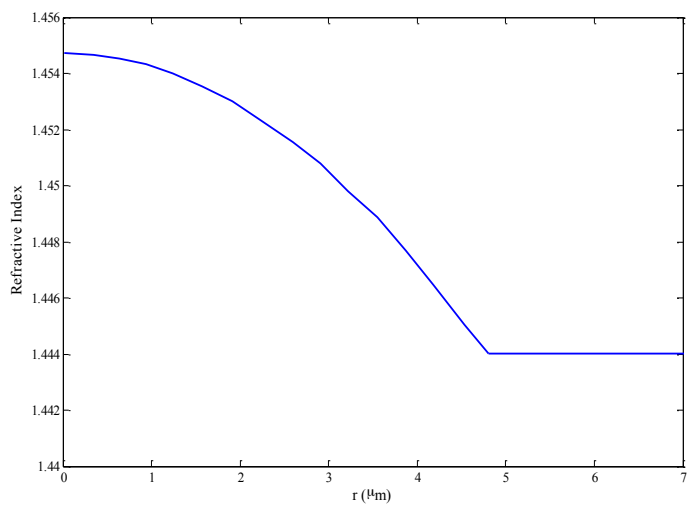

Figure 1: Refractive index profile of the designed structure.

The bending loss value of the structure at $1.55 \mu \mathrm{m}$ with 5 $\mathrm{mm}$ radius of curvature is $0.0018 \mathrm{~dB} / \mathrm{turn}$. The negligible BL is achieved owing to the introduced cost function. The bending loss behavior of the structure is illustrated in Figure 2. According to ITU-T G652.B recommendation, the bending loss value of the bend insensitive fiber at $1.55 \mu \mathrm{m}$ must be less than $0.1 \mathrm{~dB} /$ turn for $5 \mathrm{~mm}$ bending radius. The designed structure not only satisfies the desired condition at $1.55 \mu \mathrm{m}$, but also in the $\mathrm{S}+\mathrm{C}+\mathrm{L}$ communication bands. In the well-known communication bands of $\mathrm{S}, \mathrm{C}$ and $\mathrm{L}$, the bending loss is calculated to be in [3.6e-4 : 1.6e-3], [1.6e-3 : $3.8 \mathrm{e}-3]$ and [3.8e-3 : 1.1e-2] dB/turn intervals, respectively.

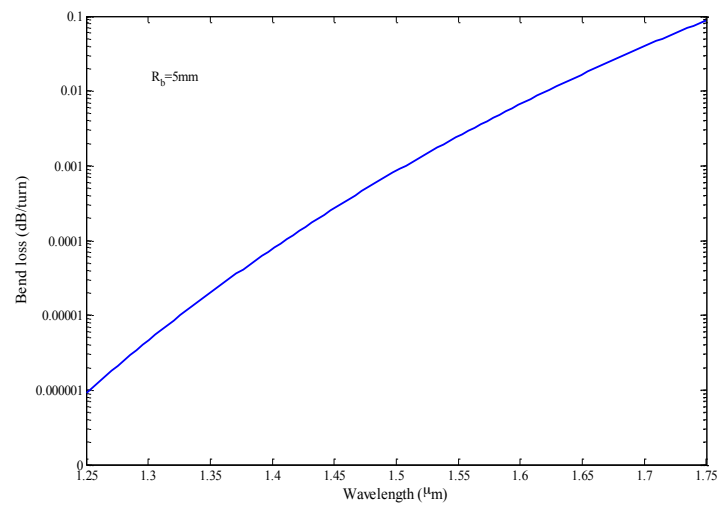

Figure 2: Bending loss (dB/turn) vs. wavelength $(\mu \mathrm{m})$ for $5 \mathrm{~mm}$ curvature radius.

The cost function of the optimization procedure has two significant terms. The first one is bending loss. The second term is responsible to reduce the sensitivity of the structure to applied external strain. Figure 3 shows the $n_{\text {eff }}$ responses to strain in the designed fiber structure. Based on the curve presented in this figure, it is obvious that application of tensile strain leads to the reduction in $n_{\text {eff }}$ value. In the meantime, this parameter increases with application of compressive strain. This response is expected regarding the results presented in [18], indeed. The strain sensitivity of the $\mathrm{n}_{\text {eff }}$ at $\lambda=1.55 \mu \mathrm{m}$ is calculated to be $-4.5 \times 10^{-8} \mu \varepsilon^{-1}$. According to Eq. 10 application of tensile and compressive 
strains result respectively in reduction and increment in the refractive index and thus the effective refractive index experiences same trends, either. It is useful to mention that the $n_{\text {eff }}$ sensitivity to strain is smaller than the results presented in [18, 22-24]. It is due to using of special cost function which enhanced the stability of the structure to strain.

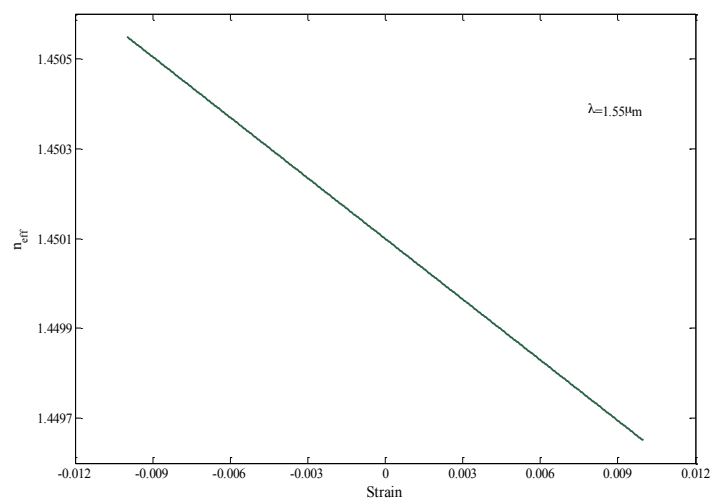

Figure 3: Effective refractive index vs. strain at $\lambda=1.55 \mu \mathrm{m}$.

The MFD is an important parameter to characterize an optical fiber and has been used extensively for conventional optical fibers. The MFD of the graded index based structure is successfully tuned to $8.53 \mu \mathrm{m}$ thanks to the design procedure. This parameter has a close relation with the electrical field distribution which is managed by effective refractive index of the structure. Any alteration in the $\mathrm{n}_{\text {eff }}$ of the structure due to applied strain could vary its MFD too. The strain impact on MFD of the considered optical fiber can be investigated, either. Figure 4 presents the variation of the MFD owing to strain at $1.55 \mu \mathrm{m}$. In this figure MFD is: $\triangle M F D=\left.M F D\right|_{\varepsilon=0}-\left.M F D\right|_{\varepsilon \neq 0}$.

Generally, MFD enlarges gradually as the applied tensile increases. Through the physical point of view, based on Figure 3, due to the tensile strain induction the effective refractive index of the structure falls. Hence, the fiber capability to bind the light reduces and the electrical field spreads more in the cladding region. In other respects, the field distribution becomes smoother and consequently the mode field diameter enlarges. The same discussion would be valid for the effect of the compressive strain on MFD. It is considerable that the effective area $\left(\mathrm{A}_{\text {eff }}\right)$ of the fiber would vary due to these changes on the electrical field distribution. The strain sensitivities of the MFD and $\mathrm{A}_{\text {eff }}$ at $1.55 \mu \mathrm{m}$ are $1.8 \mathrm{pm} \mu \varepsilon^{-1}$ and $6.3 \times 10^{-6} \mu \mathrm{m}^{2} \mu \varepsilon^{-1}$ respectively. Noting the results reported in $[18,22-24]$ the transmission characteristics of the proposed structure exhibit high stability to external strain application.

The effective area of the designed optical fiber is about $58 \mu \mathrm{m}^{2}$ which is large for bend insensitive single-mode optical fibers. Another essential concept to consider is the higher order mode cut off wavelength $\left(\lambda_{\mathrm{c}}\right)$. According to ITU-T standard for bend insensitive optical fibers, the $\lambda_{c}$ must be less than $1260 \mathrm{~nm}$ [25]. However, for the designed structure in this article, $\lambda_{\mathrm{c}}$ meets $1236 \mathrm{~nm}$ which totally conforms the mentioned standard.

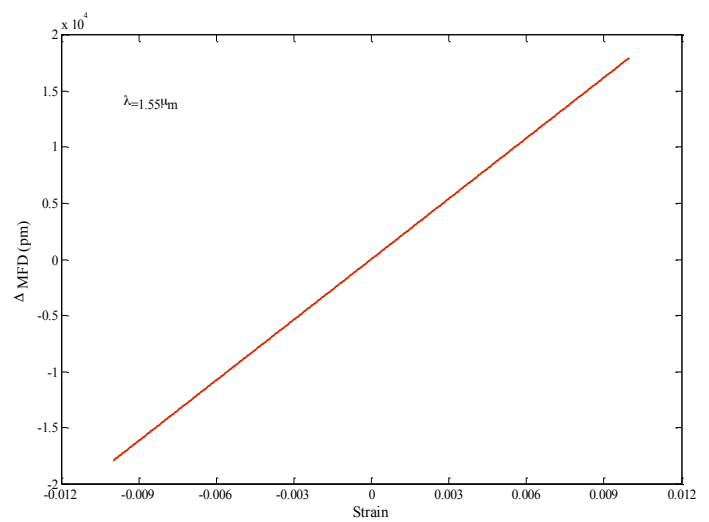

Figure 4: Mode field diameter variation (pm) vs. strain at $\lambda=1.55 \mu \mathrm{m}$.

\section{Study influence of raised outer cladding addition}

In this section, the effect of raised outer cladding placement on transmission behavior of the suggested graded index optical fiber is studied. A well-known technique for the reduction of the nonlinear effects in conventional optical fibers is the addition of raised outer clad to the structure [26]. In this method, penetration of the electrical field in the cladding region boosts the $A_{\text {eff }}$ which weakens the resistivity of the fiber to curvature. However, the BL could be restricted through augmentation of the field slope descent. The refractive index of the proposed multilayer structure is presented in Figure 5. According to the design procedure the profile of the core is extracted by the Gaussian assumption of the field distribution at the operational wavelength $\left(\lambda_{\mathrm{op}}\right)$. In order to evaluate characteristics of the structure, some optical and geometrical parameters are defined as follow:

$\Delta=\frac{n_{\max }^{2}-n_{4}^{2}}{2 n_{4}^{2}} \approx \frac{n_{\max }-n_{4}}{n_{4}}$

$R=\frac{n_{\max }-n_{3}}{n_{3}-n_{e}}$

$Q=\frac{r_{e}}{b}, \quad P=\frac{r_{e}}{c}$

where according to section $2, n_{\max }$ and $n_{e}$ are related to $n_{\text {eff }}$ and $r_{e}$. Furthermore, the $n_{3}$ and $n_{4}$ values are relevant to $n_{\max }$ and $n_{e}$ through and $\mathrm{R}$.

Based on the linear polarization approximation (LP), to calculate the electrical field distribution, the guided modes and propagating wave vectors are extracted using TMM. Employment of raised outer cladding leads to remarkable variation in the electrical field distribution manner in the cladding region. So, expectedly, the MFD management is partially failed. In other respects, the MFD value in the structure exhibited in Figure 5, depends on the field distribution not only in the core, but also in the cladding. 


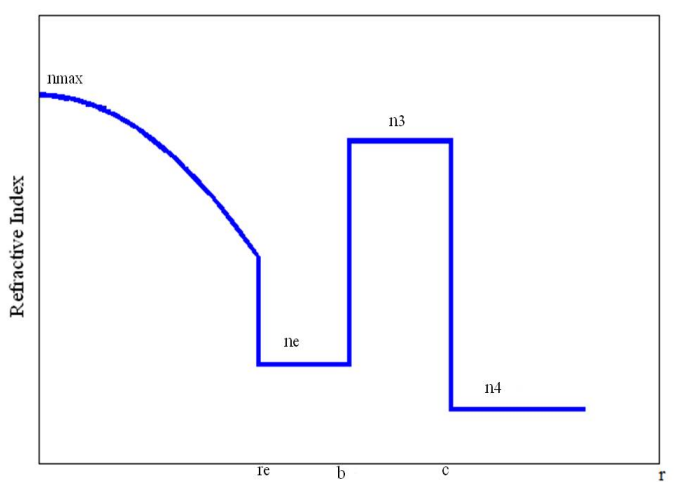

Figure 5: Refractive index profile of the suggested multiclad structure.

Exploiting the cost function presented in Eq. 11, the optical fiber, holding the refractive index profile exhibited in Figure 5 , is designed. In this case, the genes of the chromosomes in the initial population increase to six. Then by applying the defined cost function, the bending loss and strain sensitivity of the $n_{\text {eff }}$ are minimized at $1.55 \mu \mathrm{m}$. The outputs attained from optimization process are listed in Table 2 and the refractive index profile of the optimized structure is illustrated in Figure 6. Both $n_{\text {eff }}$ and $r_{e}$ are smaller than the ones presented in Table 1 . The $n_{\text {eff }}$ decrement could raise the bending loss of the multilayer structure.

Table 2: GA results for optimal structural parameters of the multilayer fiber.

\begin{tabular}{|c|c|c|c|c|c|c|}
\hline Parameter & $\mathrm{n}_{\mathrm{eff}}$ & $\mathrm{re}(\mu \mathrm{m})$ & & $\mathrm{R}$ & $\mathrm{Q}$ & $\mathrm{P}$ \\
\hline Value & 1.4487 & 3.9 & $6.2 \mathrm{e}-3$ & 1.76 & 0.75 & 0.56 \\
\hline
\end{tabular}

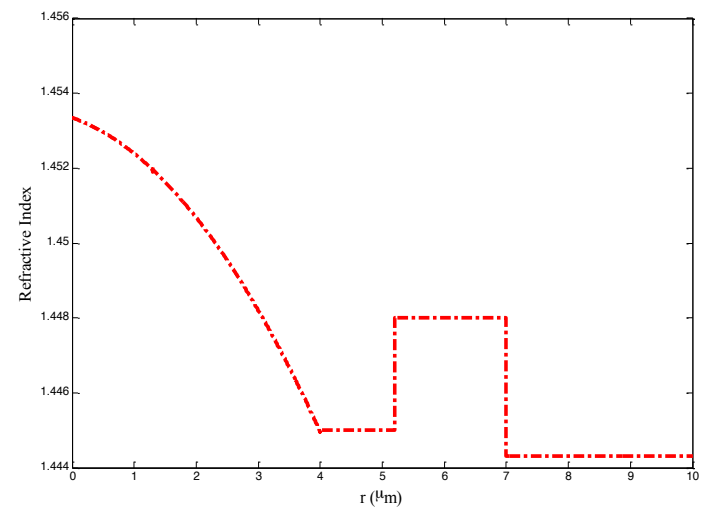

Figure 6: Refractive index profile of the designed multilayer structure.
The MFD, BL, and $\mathrm{A}_{\text {eff }}$ are $8.63 \mu \mathrm{m}, 0.0093 \mathrm{~dB} /$ turn and $87.3 \mu \mathrm{m}^{2}$, respectively. Note that, although the $\mathrm{BL}$ is increased slightly due to further leading of electrical field distribution to the out of core region, the attained $\mathrm{BL}$ is still negligible. However, there is $50 \%$ modification in $\mathrm{A}_{\text {eff }}$. Moreover, there is a boost in $n_{\text {eff }}$ sensitivity to strain which is calculated to be $-6.7 \times 10^{-8} \mu \varepsilon^{-1}$. This sensitivity is still of better stability compared to the results exhibited in $[18,22-$ 24].

Despite the noticeable difference in the refractive index profiles of the two structures, the fibers designed according to Figure 1 and Figure 6, exhibit almost the equal MFDs. Yet, the effective areas of the two structures are entirely different. Physics behind this feature is explained regarding the integration of the electrical field distributions and also their deviations. The effective area depends only on the field distribution, but the MFD depends on both the field distribution and its first deviation. Figure 7 shows a relatively proportional growth in the integration of field distribution and its deviation in the raised outer-layer which remains the MFD unchanged. The noticeable point is that the raised outer-type structure holds large effective area and bend insensitive property.

As the final issue, the $\lambda_{\mathrm{c}}$ parameter in the multilayer structure is pointed. The value is reduced to $1228 \mathrm{~nm}$ as a result of fall in the core radius.

\section{Conclusion}

The proposal of the design method for bend insensitive single-mode optical fibers appropriate for indoor wiring is presented. The employed design procedure has assured the resistivity of the structure to external strain induction. Simulation results show bending loss of $0.0018 \mathrm{~dB}$ at 1.55 $\mu \mathrm{m}$ for single turn of $5 \mathrm{~mm}$ curvature radius while the MFD and effective area are $8.53 \mu \mathrm{m}$ and $58 \mu^{2}$, respectively. The strain sensitivity of $n_{\text {eff }}$ is calculated to be $-4.5 \times 10^{-8} \mu \varepsilon^{-}$ 1. The impact of adding raised outer-clad has been investigated, either. Simulation outcomes admit that the designed graded index optical fiber with raised outer-clad structure is the ultra large effective area bend insensitive optical fiber. The values of bending loss for single turn of $5 \mathrm{~mm}$ curvature radius, effective area, and MFD are, respectively, $0.0093 \mathrm{~dB}, 87 \mu \mathrm{m}^{2}$ and $8.63 \mu \mathrm{m}$ at $1.55 \mu \mathrm{m}$ operational wavelength. The changes in $\mathrm{n}_{\mathrm{eff}}$ due to variation in applied strain is $-6.7 \times 10^{-8} \mu \varepsilon^{-1}$ which is still of satisfactory stability. 

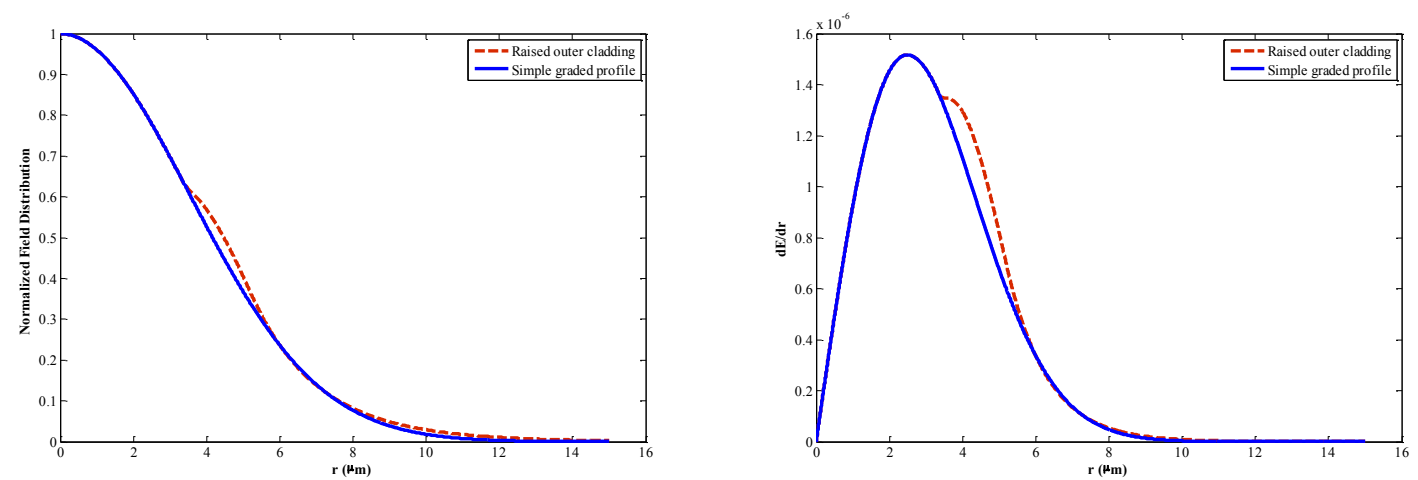

Figure 7: Normalized field distributions and their first deviation in simple graded index profile and raised outer-clad structures.

\section{References}

[1] D.S. Burpee, P.W. Shumate, Emerging residential broadband telecommunications, Proceeding IEEE 82:604614, 1994.

[2] P.W. Shumate, Optical fibers reach into homes, IEEE Spectrum 26:43-47, 1989.

[3] P. Weimann, T. Goddard, D. Mazzarese, K. Konstadinidis, Design considerations for next-generation optical drop cables using bend optimized fiber, the 56th IWCS, Florida, USA. pp. 489-494, November 11-14 2007.

[4] J. Cai, Y. Ishikawa, K. Wada, Strain induced bandgap and refractive index variation of silicon, Optics Express 21:7162-7170, 2013.

[5] J. Castrellon, Fiber Optic Sensors. In Optical Fiber Sensors: An Overview, Yasin M, editor. InTech, pp. 112139, 2012.

[6] A. Ghatak, K. Thyagarajan, Introduction to fiber optics, 3rd ed.; Cambridge University Press, 2002.

[7] K. Ichii, N. Yamada, M. Fujimaki, K. Harada, K. Tsurusaki, Characteristics of low macrobending-loss SMF (FutureGuide-SR15E) with low water peak, IEICE General Conference, Tokyo, Japan, paper B-10-2, 2004.

[8] S. Matsuo, T. Nunome, T. Yoshita, T. Hamada, K. Himeno, Design optimization of trench index profile for the same dispersion characteristics with SMF, OFC/NFOEC, Anaheim, CA, USA, paper JWA2, 25-29 March 2007.

[9] J.C. Knight, T.A. Briks, R.F. Cregan, P.S. Russell, J.P. De Sandro, Large mode area photonic crystal fibers, Electronics Letters 34:1347-1348, 1998.

[10] K. Miyake, M. Hachiwaka, T. Kinoshita, S. Yamaguchi, H. Kubota, S. Kawanishi, Bend resistant photonic crystal fiber compatible with conventional singlemode fiber, ECOC, Stockholm, Sweden. pp.Mo3.3.4, 5-9 September 2004.

[11] F. Poli, A. Cucinotta, S. Selleri, Photonic Crystal Fibers, properties and applications, 1st ed.; springer, 2007.

[12] D. Marcuse, Gaussian approximation of the fundamental modes of graded-index fibers, Optical Society of America 68:103-109, 1978.
[13] A. Rostami and S. Makouei, Novel design method for single-mode bend insensitive fiber appropriate for FTTH application, Fiber and Integrated Optics 30: 102-111, 2011.

[14] A. Rostami and S. Makouei, Novel low-bend large effective area fiber for fiber-to-the-home application, Fiber and Integrated Optics 30: 1-8, 2011.

[15] M. Shenoy, K. Thygarajan, A. Ghatak, Numerical analysis of optical fibers using matrix approach, Lightwave Technology 6: 1285-1291, 1988.

[16] J.I. Sakai, T. Kimura, Bending loss of propagation modes in arbitrary index profile optical fibers, Applied Optics 17:1499-1506, 1978.

[17] D. Marcuse, Curvature Loss Formula for Optical Fibers, Optical Society of America 66: 216-220, 1976.

[18] F. Makouei, S. Makouei, GA based management of strain response in RII-type single-mode optical fiber, Optik 127:8333-8340, 2016.

[19] T. Baeck, F. Hoffmeister, H.P. Schwefel, An Overview of Evolutionarv Algorithms for Parameter Optimization, Evolutionary Computation 1:1-24, 1993.

[20] P.K. Bachmann, W. Hermann, H. Wehr, D.U. Wiechert, Stress in optical waveguide. 2: Fibers, Applied Optics 26:1175-1182, 1987.

[21] B. Kim, Y. Park, D. Kim, U. Paek, W. Han, Observation and analysis of residual stress development resulting from $\mathrm{OH}$ impurity in optical fibers, Optics Letters 27: 806-808, 2002.

[22] S. Makouei, F. Makouei, Strain Effect Study on Mode Field Diameter and Effective Area of WII Type Single Mode Optical Fiber, Advanced Electromagnetics 5:53-61, 2016.

[23] F. Makouei and S. Makouei, Design of temperature insensitive in vivo strain sensor using multilayer singlemode optical fiber, Frontiers of Optoelectronics 9: 621626, 2016.

[24] S. Makouei and F. Makouei, Thermal stability enhanced ZDSF proposal for ultra high-speed long haul communication systems, Optics Communications 388: 1220, 2017.

[25] ITU Recomandations, G.652, G.657 
[26] M. Savadi-Oskouei, S. Makouei, A. Rostami, Z.D. Koozeh-kanani, Proposal for optical fiber designs with ultrahigh effective area and small bending loss applicable to long haul communications, Applied Optics 46: 63306339, 2007. 